\title{
Thrombocytopenia in patients with melanoma receiving immune checkpoint inhibitor therapy
}

Eileen Shiuan ${ }^{1,2 \dagger}$, Kathryn E. Beckermann ${ }^{3 *}$, Alpaslan Ozgun ${ }^{4}$, Ciara Kelly ${ }^{5}$, Meredith McKean ${ }^{6}$, Jennifer McQuade , Mary Ann Thompson ${ }^{11}$, Igor Puzanov ${ }^{12}$, John P. Greer ${ }^{3}$, Suthee Rapisuwon ${ }^{8}$, Michael Postow ${ }^{9,10}$,

Michael A. Davies ${ }^{7}$, Zeynep Eroglu ${ }^{4}$ and Douglas Johnson ${ }^{3}$

\begin{abstract}
Background: Immune checkpoint inhibitors, including antibodies against programmed death 1 (PD-1) and cytotoxic T-lymphocyte antigen 4 (CTLA-4), are being used with increasing frequency for the treatment of cancer. Immune-related adverse events (irAEs) including colitis, dermatitis, and pneumonitis are well described, but less frequent events are now emerging with larger numbers of patients treated. Herein we describe the incidence and spectrum of thrombocytopenia following immune checkpoint inhibitor therapy and two severe cases of idiopathic thrombocytopenic purpura (ITP).

Case presentations: A 47-year-old female with recurrent BRAF mutant positive melanoma received combination anti-PD-1 and anti-CTLA-4. Two weeks later, she presented with mucosal bleeding, petechiae, and thrombocytopenia and was treated with standard therapy for ITP with steroids and intravenous immunoglobulin (IVIG). Her diagnosis was confirmed with bone marrow biopsy, and given the lack of treatment response, she was treated with rituximab. She began to have recovery and stabilization of her platelet count that ultimately allowed her to be retreated with PD-1 inhibition with no further thrombocytopenia. A second patient, a 45-year-old female with a BRAF wild-type melanoma, received anti-PD-1 monotherapy and became thrombocytopenic 43 days later. Three weeks of steroid treatment improved her platelet count, but thrombocytopenia recurred and required additional steroids. She later received anti-CTLA-4 monotherapy and developed severe ITP with intracranial hemorrhage. Her ITP resolved after treatment of prednisone, IVIG, and rituximab and discontinuation of checkpoint inhibition. In a retrospective chart review of 2360 patients with melanoma treated with checkpoint inhibitor therapy, $<1 \%$ experienced thrombocytopenia following immune checkpoint inhibition, and of these, most had spontaneous resolution and did not require treatment.

Conclusions: Thrombocytopenia, especially ITP, induced by immune checkpoint inhibitors appears to be an uncommon irAE that is manageable with observation in mild cases and/or standard ITP treatment algorithms. In our series, the majority of patients had mild thrombocytopenia that resolved spontaneously or responded to standard corticosteroid regimens. However, in two severe cases, IVIG and rituximab, in addition to steroids, were required. Checkpoint inhibition was resumed successfully in the first patient but rechallenge was not tolerated by the second patient.
\end{abstract}

Keywords: Checkpoint inhibitor, PD-1, CTLA-4, Thrombocytopenia, Immune thrombocytopenic purpura, Melanoma

\footnotetext{
* Correspondence: katy.beckermann@vanderbilt.edu

${ }^{\dagger}$ Equal contributors

${ }^{3}$ Division of Hematology/Oncology, Vanderbilt University Medical Center, 777

PRB, 2220 Pierce Ave, Nashville, TN, USA

Full list of author information is available at the end of the article
} 


\section{Background}

Immune checkpoint inhibitors, including antibodies against programmed death 1 (PD-1) receptor, are quickly becoming a staple in our arsenal of anti-cancer agents. PD-1 signaling normally inactivates effector T cells when bound to its ligands PD-L1 and PD-L2; thus, inhibition of this pathway reinvigorates $\mathrm{T}$ cell antitumor responses [1]. Nivolumab and pembrolizumab are monoclonal antibodies that block PD-1 and have demonstrated substantial benefit in melanoma, non-small cell lung cancer, renal cell carcinoma, Hodgkin lymphoma, and numerous other cancers [2].

Although inhibitors of PD-1 provide significant therapeutic benefit, many immune-related adverse events (irAEs) have emerged with these therapies. The most common irAEs include dermatologic toxicities and thyroid dysfunction [3-5]. Other clinically significant toxicities include colitis, hypophysitis, pneumonitis, and hepatitis [4-7]. These events arise from dysregulation of self-tolerance that is normally mediated by PD-1/PD-L1 interactions [8]. Additionally, anti-PD-1 (specifically nivolumab) may be combined with ipilimumab, an antibody against cytotoxic T-lymphocyte antigen 4 (CTLA-4), which when bound to costimulatory molecules on antigen-presenting cells inactivates $\mathrm{T}$ cells. Ipilimumab works synergistically with anti-PD-1 agents and improves antitumor efficacy but also increases the frequency and severity of irAEs [3, 5].

Recently, cases of hematologic irAEs were reported with anti-PD-1 therapy, specifically autoimmune hemolytic anemia and immune thrombocytopenic purpura (ITP) [9-12]. Although cases of thrombocytopenia induced by either pembrolizumab or ipilimumab alone have been reported, its incidence, spectrum of severity, and development of ITP have not been established [13, 14]. Given the rapid rise of immunomodulatory therapy use in numerous cancers, there is a clear need to identify and characterize these hematologic irAEs. Here, we report two cases of severe ITP resulting from checkpoint inhibitor therapy and the largest multi-institutional case series of thrombocytopenia induced by checkpoint inhibitor therapy.

\section{Case presentations}

A 47-year-old patient presented in 2011 with stage IIb melanoma on her left forearm, which was removed by wide local excision with a concurrent negative sentinel lymph node biopsy. Four years later, she presented with recurrent metastatic melanoma with a $B R A F^{\mathrm{V} 600 \mathrm{M}}$ mutation. She initially responded to combination BRAF and MEK inhibition but developed progressive disease within five months. Two weeks later, she received her first infusion of combination ipilimumab and nivolumab. She developed bleeding from mucosal areas and petechiae fifteen days following her first dose of combination checkpoint inhibitor therapy with severe thrombocytopenia $(\mathrm{PLT}<5000 / \mathrm{uL})$ and an elevated immature platelet fraction of $15.4 \%$ ( 0.9 to $7.0 \%$ normal range). She had no history of autoimmune or coagulation disorders, and the remainder of her laboratory evaluation was unrevealing. She was presumed to have new-onset ITP and was started on methylprednisolone and intravenous immunoglobulin (IVIG) (Fig. 1). After five days of steroids and IVIG without significant improvement in her platelet count, a bone marrow biopsy revealed a hypercellular marrow with increased megakaryocytes (Fig. 2), further supporting the diagnosis of ITP. Her treatment was escalated to weekly rituximab with addition of a single dose of romiplostim, a thrombopoietin analog. Seven days after steroids and IVIG, and two days after rituximab, her platelet counts began to improve, reaching normal range $(137,000$ to $397,000 / \mathrm{uL}$ ) by one week, and she subsequently did not require additional platelet transfusions (Fig. 1). Between her second and third infusion of rituximab, she transiently relapsed to a platelet count of $54,000 / \mathrm{uL}$. She received a total of four doses of rituximab in accordance with standard therapy for ITP. Eight days after her last dose, she was rechallenged with nivolumab monotherapy and subsequently experienced a partial response with no relapse of her ITP.

A second patient, a 45-year-old female with a BRAF wild-type melanoma, received nivolumab as neoadjuvant therapy in a clinical trial and became thrombocytopenic $(49,000 / \mathrm{uL}) 43$ days later. She was asymptomatic and was treated with prednisone for three weeks, with elevation of platelet levels to baseline $(307,000 / \mathrm{uL})$. However, thrombocytopenia recurred with a platelet nadir of $28,000 / \mathrm{uL}$, and she required an additional 12-week steroid taper before her platelets recovered. She later developed metastatic disease to the brain and underwent resection of a large frontal lesion, as well as gamma knife irradiation to several smaller lesions. Due to limited treatment options, she was administered ipilimumab monotherapy, eight months after receiving nivolumab. Within eight days, her platelet count decreased from 75,000 to $8000 / \mathrm{uL}$, and two days later, she developed hemorrhage in her intracranial metastasis with no detectable platelets. Her ITP ultimately resolved after treatment with prednisone, IVIG, and rituximab and discontinuation of ipilimumab.

To assess the incidence and clinical patterns of thrombocytopenia, including potential cases of ITP, following immune checkpoint inhibition, we performed retrospective reviews of electronic medical records at Georgetown Lombardi Cancer Center, Memorial Sloan Kettering Cancer Center, Moffitt Cancer Center, MD Anderson Cancer Center, and Vanderbilt-Ingram Cancer Center. Patients with melanoma were included if they experienced thrombocytopenia following treatment with 


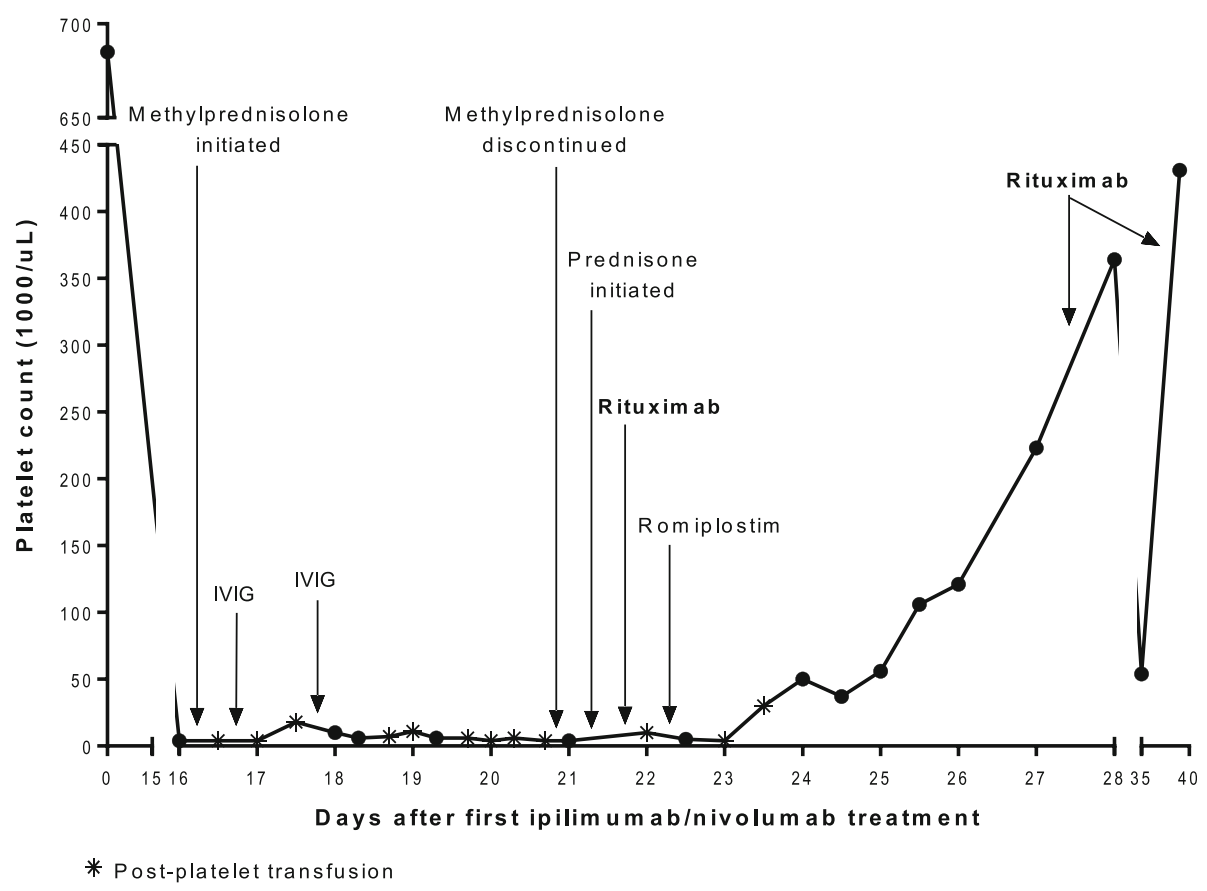

Fig. 1 Checkpoint inhibitor-induced ITP refractory to glucocorticoids subsequently responds to second-line treatment

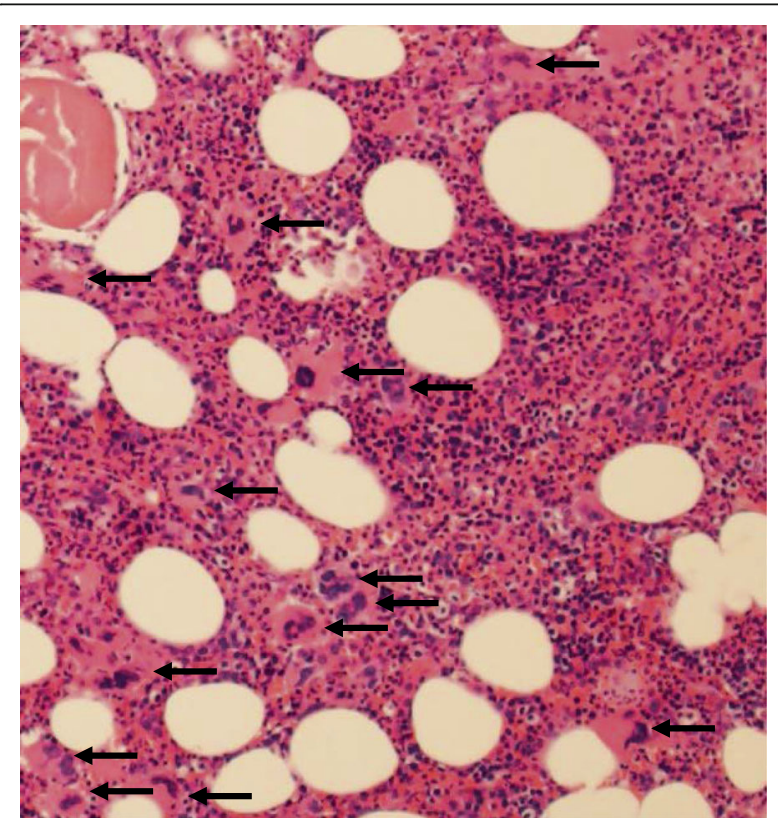

Fig. 2 Bone marrow from patient with checkpoint inhibitor-induced ITP before rituximab treatment. H\&E stained section of the bone marrow biopsy, $100 \times$ magnification. The bone marrow is moderately hypercellular for age with trilineage hematopoiesis and increased megakaryocytes (black arrows) with a range of morphologies and mild clustering. These findings, coupled with the patient's peripheral thrombocytopenia and elevated IPF, are compatible with a diagnosis of ITP a checkpoint inhibitor that was clinically diagnosed as ITP or was not attributable to another cause. The project was approved by IRBs of respective institutions with waiver of consent. Statistical analysis was performed using $\mathrm{R}$ version 3.3.0.

We assessed the frequency of thrombocytopenia induced by treatment with checkpoint inhibitors across these five institutions and identified 11 cases, several presumed to be ITP based on clinical diagnostic criteria. A total of 2360 patients with melanoma receiving checkpoint inhibitor therapy were reviewed, suggesting an incidence of well under $1 \%$. These patients were Caucasian men (58\%) or women (42\%) with melanoma; none had a previous diagnosis of ITP or a history of thrombocytopenia prior to initiation of treatment. Various checkpoint inhibitor regimens were represented (Table 1). The average time to onset of checkpoint inhibitor-induced thrombocytopenia was 70 days (range, 12 to 173 days), and the average platelet count was $61,000 / \mathrm{uL}$ (range, $<5000$ to $104,000 / \mathrm{uL}$ ) with an average decrease of $70 \%$ from baseline (range, 38 to $99 \%$ ). No significant differences were found among the varying checkpoint inhibitor regimens.

Of the 11 patients, four required immunosuppressive treatment with corticosteroids, and two of those cases were refractory to steroids. A higher percentage of patients treated with ipilimumab (single agent or combined with nivolumab) required immunosuppressive treatment $(75 \%, 3$ of 4$)$ compared to those treated with anti-PD-1 monotherapy (14\%, 1 of 7$)$. The majority of 


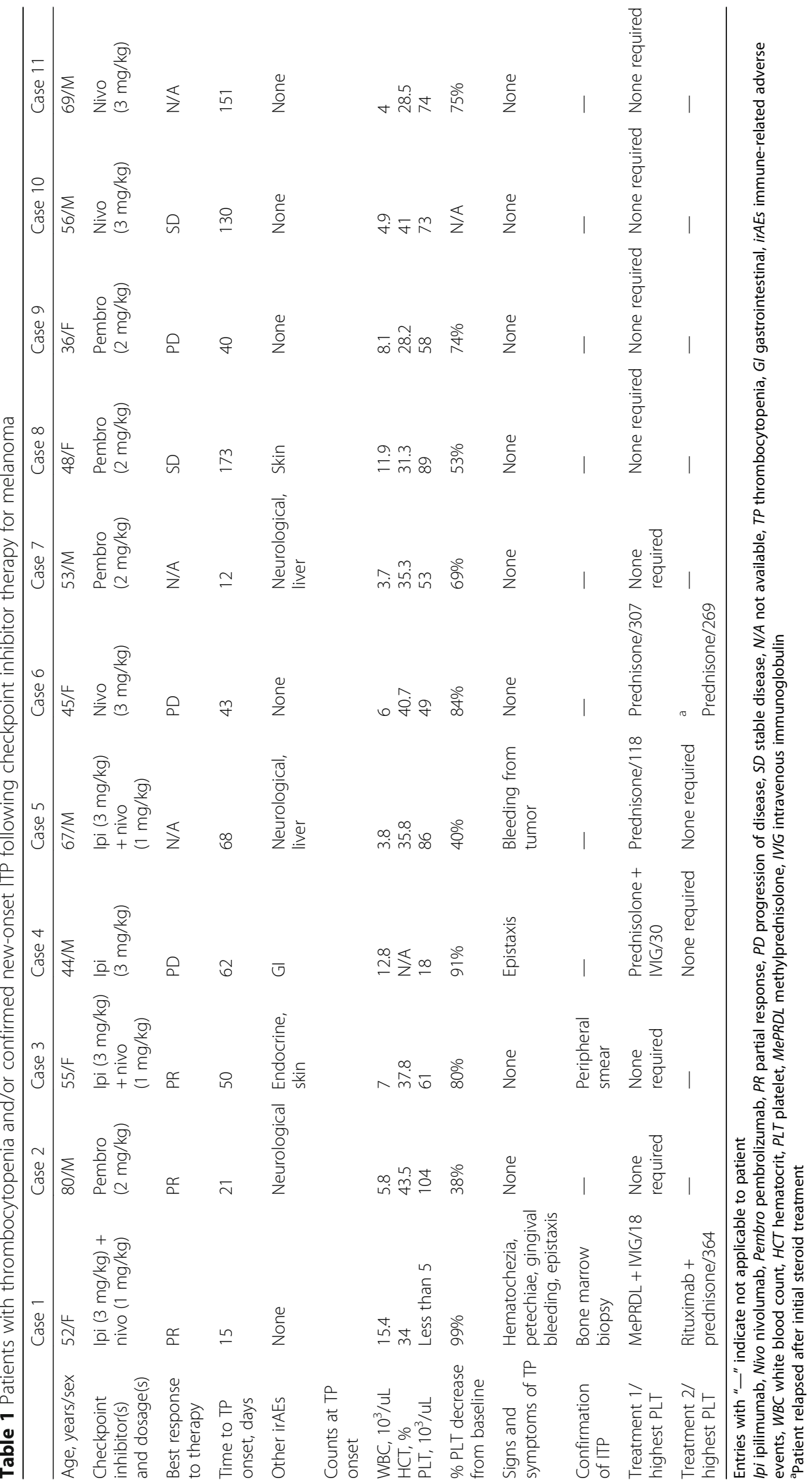


patients displayed no clinical signs or symptoms of thrombocytopenia and required no therapies with spontaneous resolution (Table 1). Our first case described in detail above had the most severe episode of thrombocytopenia with confirmed ITP by bone marrow biopsy.

\section{Conclusions}

Thrombocytopenia, especially ITP, induced by immune checkpoint inhibitors appears to be a relatively uncommon irAE that is manageable with standard treatment algorithms. In our series, the majority of patients had mild thrombocytopenia that resolved spontaneously or responded to standard corticosteroid regimens. However, in two severe cases, steroids, IVIG, and rituximab were administered with ultimate recovery. In the first case, nivolumab monotherapy was resumed with excellent tolerance. On the other hand, the second patient relapsed with subsequent immune checkpoint inhibition.

Primary ITP is a disorder caused by the formation of autoantibodies targeting platelet antigens, leading to thrombocytopenia [15]. ITP is a diagnosis of exclusion and may be challenging given the lack of specific testing and a wide differential diagnosis. ITP is thought to occur after an inciting event activates or alters the immune system, such as an infection, hematopoietic malignancy, or pharmacologic immune checkpoint inhibition [16]. However, most cases are idiopathic in etiology. A majority of acute cases (50-90\%) are responsive to standard corticosteroid and IVIG therapy, though a fraction of cases require second-line treatment, usually involving a combination of rituximab and a thrombopoietin agonist [17]. In mouse models, there is loss of peripheral selftolerance through alteration of immune homeostasis and evidence of regulatory $\mathrm{T}$ cell (Treg) deficiency associated with ITP [18]. Comparison of bone marrow between patients with ITP and normal donors revealed that those with ITP have lower levels of Tregs and abnormal levels of Th1 and Th17 cells [16]. Recent work demonstrated that patients with chronic ITP exhibit lower levels of PD-1 expression in total peripheral blood samples, compared with healthy controls $[19,20]$. A single case report showed that a patient who developed nivolumabinduced ITP had higher PD-1 expression on B cells [11].

Our experience suggests that thrombocytopenia, including ITP, may rarely complicate immune checkpoint inhibitor therapy but is usually mild and can resolve spontaneously or with standard treatment algorithms. The onset of ITP varies substantially, though a majority occurs within the first 12 weeks after initiation of checkpoint inhibition, consistent with other irAEs [21-23]. Although our observations on checkpoint inhibitor rechallenge after resolution of ITP are limited, our experience suggests that increased clinical vigilance should be used, especially with ipilimumab.

\section{Abbreviations}

CTLA-4: Cytotoxic T-lymphocyte antigen 4; irAE: Immune-related adverse event; ITP: Immune thrombocytopenic purpura; IVIG: Intravenous immunoglobulin; PD-1: Programmed death 1; PD-L1: Programmed death ligand 1; PD-L2: Programmed death ligand 2; Treg: Regulatory T cell

\section{Acknowledgements}

The authors would like thank the patients presented in this study.

\section{Funding}

This work was supported by funding from the National Institutes of Health (T32 GM0734, E.S. and K23 CA204726, D.J.), the Merck-Cancer Research Institute Irvington fellowship (K.B.), and the Brock Endowment fellowship (K.B.).

\section{Availability of data and materials}

All data generated or analyzed during this study are included in this article.

\section{Authors' contributions}

E.S. and K.B. collected and analyzed the data and wrote the manuscript; E.S. created the figures and table; K.B. coordinated data collection from outside institutions and IRB approvals; E.S., K.B., C.K., A.O., Z.E., M.M., J.M., and S.R. collected patient data; M.A. evaluated bone marrow biopsy; D.J. provided expertise and wrote and edited the manuscript. All authors read and approved the manuscript.

\section{Competing interests}

D.J. is on advisory boards for Bristol Myers Squibb and Genoptix and receives research funding from Incyte.

\section{Consent for publication}

A general research consent was signed by study participants to cover the identifying data in the manuscript, including Table 1.

\section{Ethics approval and consent to participate}

This work was approved by IRBs of all contributing institutions with waiver of consent, including the Vanderbilt University Medical Center IRB, Memorial Sloan Kettering Cancer Center IRB, Georgetown University Medical Center IRB, Moffitt Cancer Center IRB, and MD Anderson IRB.

\section{Author details}

${ }^{1}$ Medical Scientist Training Program, Vanderbilt University Medical Center, Nashville, TN 37232, USA. ${ }^{2}$ Department of Cancer Biology, Vanderbilt University Medical Center, Nashville, TN 37232, USA. ${ }^{3}$ Division of Hematology/Oncology, Vanderbilt University Medical Center, 777 PRB, 2220 Pierce Ave, Nashville, TN, USA. ${ }^{4}$ Department of Cutaneous Oncology, Moffitt Cancer Center and Research Institute, Tampa, FL 33612, USA. ${ }^{5}$ Sarcoma

Oncology Service, Department of Medicine, Memorial Sloan Kettering Cancer Center, New York, NY 10065, USA. ${ }^{6}$ Division of Cancer Medicine, University of Texas MD Anderson Cancer Center, Houston, TX 77030, USA. ${ }^{7}$ Melanoma Medical Oncology, University of Texas MD Anderson Cancer Center, Houston, TX 77030, USA. ${ }^{8}$ Division of Hematology/Oncology, Lombardi Comprehensive Cancer Center, Georgetown University, Washington, DC 20057, USA. ${ }^{9}$ Melanoma and Immunotherapeutics Service, Department of Medicine, Memorial Sloan Kettering Cancer Center, New York, NY 10065,

USA. ${ }^{10}$ Weill Cornell Medical College, Cornell University, New York, NY 10065, USA. "'Department of Pathology, Microbiology, and Immunology, Vanderbilt University Medical Center, Nashville, TN 37232, USA. ${ }^{12}$ Roswell Park Cancer Institute, Buffalo, NY 14263, USA.

Received: 2 November 2016 Accepted: 6 January 2017

Published online: 21 February 2017

\section{References}

1. Chen L, Han X. Anti-PD-1/PD-L1 therapy of human cancer: past, present, and future. J Clin Invest. 2015;125:3384-91.

2. Wolchok JD. PD-1 blockers. Cell. 2015;162:937.

3. Larkin J, Chiarion-Sileni V, Gonzalez R, Grob JJ, Cowey CL, Lao CD, Schadendorf D, Dummer R, Smylie M, Rutkowski P, et al. Combined Nivolumab and Ipilimumab or Monotherapy in Untreated Melanoma. N Engl J Med. 2015;373:23-34. 
4. Garon EB, Rizvi NA, Hui R, Leighl N, Balmanoukian AS, Eder JP, Patnaik A, Aggarwal C, Gubens M, Horn L, et al. Pembrolizumab for the treatment of non-small-cell lung cancer. N Engl J Med. 2015;372:2018-28.

5. Robert C, Schachter J, Long GV, Arance A, Grob JJ, Mortier L, Daud A, Carlino MS, McNeil C, Lotem M, et al. Pembrolizumab versus Ipilimumab in advanced Melanoma. N Engl J Med. 2015;372:2521-32.

6. Brahmer J, Reckamp KL, Baas P, Crinò L, Eberhardt WEE, Poddubskaya E, Antonia S, Pluzanski A, Vokes EE, Holgado E, et al. Nivolumab versus docetaxel in advanced squamous-cell non-small-cell lung cancer. N Engl J Med. 2015;373:123-35.

7. Motzer RJ, Escudier B, McDermott DF, George S, Hammers HJ, Srinivas S, Tykodi SS, Sosman JA, Procopio G, Plimack ER, et al. Nivolumab versus everolimus in advanced renal-cell carcinoma. N Engl J Med. 2015;373:1803-13.

8. Francisco LM, Sage PT, Sharpe AH. The PD-1 pathway in tolerance and autoimmunity. Immunol Rev. 2010;236:219-42.

9. Le Roy A, Kempf E, Ackermann F, Routier E, Robert C, Turpin A, Marabelle A, Mateus C, Michot J-M, Lambotte O. Two cases of immune thrombocytopenia associated with pembrolizumab. Eur J Cancer. 2016;54:172-4.

10. Kong BY, Micklethwaite KP, Swaminathan S, Kefford RF, Carlino MS. Autoimmune hemolytic anemia induced by anti-PD-1 therapy in metastatic melanoma. Melanoma Res. 2016;26(2):202-4.

11. Kanameishi S, Otsuka A, Nonomura Y, Fujisawa A, Endo Y, Kabashima K. Idiopathic thrombocytopenic purpura induced by nivolumab in a metastatic melanoma patient with elevated PD-1 expression on B cells. Ann Oncol. 2016;27:546-7.

12. Bagley SJ, Kosteva JA, Evans TL, Langer CJ. Immune thrombocytopenia exacerbated by nivolumab in a patient with non-small-cell lung cancer. Cancer Treat Commun. 2016;6:20-3.

13. Kopecký J, Trojanová P, Kubeček O, Kopecký O. Treatment possibilities of ipilimumab-induced thrombocytopenia—case study and literature review. Jpn J Clin Oncol. 2015;45:381-4.

14. Sajjad MZ, George T, Weber JS, Sokol L. Thrombocytopenia associated with ipilimumab therapy of advanced melanoma at a single institution. ASCO Meet Abstr. 2013;31:9072

15. Provan D, Newland AC. Current management of primary immune thrombocytopenia. Adv Ther. 2015;32:875-87.

16. Song $Y$, Wang $Y$ - $T$, Huang $X-J$, Kong $Y$. Abnormalities of the bone marrow immune microenvironment in patients with immune thrombocytopenia. Ann Hematol. 2016;95:959-65.

17. Cines DB, Bussel JB. How I treat idiopathic thrombocytopenic purpura (ITP). Blood. 2005;106:2244-51.

18. Aslam R, Hu Y, Gebremeskel S, Segel GB, Speck ER, Guo L, Kim M, Ni H, Freedman J, Semple JW. Thymic retention of CD4 + CD25 + FoxP3+ T regulatory cells is associated with their peripheral deficiency and thrombocytopenia in a murine model of immune thrombocytopenia. Blood 2012:120:2127-32.

19. Zhong J, Chen S, Xu L, Lai J, Liao Z, Zhang T, Yu Z, Lu Y, Yang L, Wu X, et al. Lower expression of PD-1 and PD-L1 in peripheral blood from patients with chronic ITP. Hematology. 2016;21(9):552-57.

20. Birtas Atesoglu E, Tarkun P, Demirsoy ET, Geduk A, Mehtap O, Batman A, Kaya F, Cekmen MB, Gulbas Z, Hacihanefioglu A. Soluble programmed death 1 (PD-1) is decreased in patients with immune thrombocytopenia (ITP): potential involvement of PD-1 pathway in ITP immunopathogenesis. Clin Appl Thromb Hemost. 2016;22:248-51.

21. Weber JS, Kähler KC, Hauschild A. Management of immune-related adverse events and kinetics of response with ipilimumab. J Clin Oncol. 2012;30:2691-7.

22. Michot JM, Bigenwald C, Champiat S, Collins M, Carbonnel F, Postel-Vinay S, Berdelou A, Varga A, Bahleda R, Hollebecque A, et al. Immune-related adverse events with immune checkpoint blockade: a comprehensive review. Eur J Cancer. 2016:54:139-48.

23. Friedman CF, Proverbs-Singh TA, Postow MA. Treatment of the immune-related adverse effects of immune checkpoint inhibitors: A review. JAMA Oncol. 2016;2(10):1346-353.

\section{Submit your next manuscript to BioMed Central and we will help you at every step:}

- We accept pre-submission inquiries

- Our selector tool helps you to find the most relevant journal

- We provide round the clock customer support

- Convenient online submission

- Thorough peer review

- Inclusion in PubMed and all major indexing services

- Maximum visibility for your research

Submit your manuscript at www.biomedcentral.com/submit
Biomed Central 